\title{
STRONG LARGE DEVIATION AND LOCAL LIMIT THEOREMS ${ }^{1}$
}

\author{
By NARAsinga Rao Chaganty ${ }^{2}$ and JAYARAm Sethuraman ${ }^{3}$
}

\section{Old Dominion University and Florida State University}

Most large deviation results give asymptotic expressions for $\log P\left(Y_{n} \geq\right.$ $\left.y_{n}\right)$, where the event $\left\{Y_{n} \geq y_{n}\right\}$ is a large deviation event, that is, $P\left(Y_{n} \geq y_{n}\right)$ goes to 0 exponentially fast. We refer to such results as weak large deviation results. In this paper we obtain strong large deviation results for arbitrary random variables $\left\{Y_{n}\right\}$, that is, we obtain asymptotic expressions for $P\left(Y_{n} \geq y_{n}\right)$, where $\left\{Y_{n} \geq y_{n}\right\}$ is a large deviation event. These strong large deviation results are obtained for lattice valued and nonlattice valued random variables and require some conditions on their moment generating functions. These results strengthen existing results which apply mainly to sums of independent and identically distributed random variables.

Since $Y_{n}$ may not possess a probability density function, we consider the function $q_{n}\left(y ; b_{n}, S\right)=\left[\left(b_{n} / \mu(S)\right) P\left(b_{n}\left(Y_{n}-y\right) \in S\right)\right]$, where $b_{n} \rightarrow \infty, \mu$ is the Lebesgue measure on $R$, and $S$ is a measurable subset of $R$ such that $0<\mu(S)<\infty$. The function $q_{n}\left(y ; b_{n}, S\right)$ is the p.d.f. of $Y_{n}+Z_{n}$, where $Z_{n}$ is uniform on $-S / b_{n}$, and will be called the pseudodensity function of $Y_{n}$. By a local limit theorem we mean the convergence of $q_{n}\left(y_{n} ; b_{n}, S\right)$ as $n \rightarrow \infty$ and $y_{n} \rightarrow y^{*}$. In this paper we obtain local limit theorems for arbitrary random variables based on easily verifiable conditions on their characteristic functions. These local limit theorems play a major role in the proofs of the strong large deviation results of this paper. We illustrate these results with two typical applications.

1. Introduction. The establishment of a limit distribution for a sequence of random variables $\left\{Y_{n}, n \geq 1\right\}$ provides an approximation to $P\left(Y_{n} \geq y\right)$. However, there are other aspects relating to the distribution of $Y_{n}$ for which one often desires an approximation. This could be $P\left(Y_{n} \geq y_{n}\right)$, known in the literature as a large deviation, especially when it tends to 0 exponentially fast. Another example is $f_{n}\left(y_{n}\right)$, the probability density function (p.d.f.) of $Y_{n}$ at $y_{n}$. The term, a large deviation local limit result for $Y_{n}$, is used when an asymptotic expression is established for $f_{n}\left(y_{n}\right)$ and $y_{n}$ is in the range of a large deviation for $Y_{n}$. Still another example is the pseudodensity function of $Y_{n}$, $q_{n}\left(y ; b_{n}, S\right)$, which is available even when $Y_{n}$ does not possess a p.d.f. and is defined as the p.d.f. of $Y_{n}+Z_{n}$, where $Z_{n}$ is uniform on $-S / b_{n}$. Such a result will be referred to as a local limit result for $Y_{n}$. This paper will deal with strong large deviation and local limit theorems for arbitrary random variables.

Received July 1986; revised March 1992.

${ }^{1}$ The United States government is authorized to reproduce and distribute reprints for governmental purposes notwithstanding any copyright notation thereon.

${ }^{2}$ Partially supported by the U.S. Army Research Office Grant DAAL 03-91-G-0179.

${ }^{3}$ Partially supported by the U.S. Army Research Office Grant DAAL 03-90-G-0103.

AMS 1991 subject classifications. 60F10, 60F05, 60F15.

Key words and phrases. Large deviations, local limit theorems. 
The theory of large deviations for sums of independent and identically distributed (i.i.d.) random variables and its many generalizations has a long history [see, e.g., Cramér (1938), Chernoff (1952), Ellis (1984) and Varadhan (1984)]. However, most of these results give asymptotic expressions for $\log P\left(Y_{n} \geq y_{n}\right)$ and so we choose to call them weak large deviation results. For arbitrary random variables $T_{n}$ and $Y_{n}=T_{n} / a_{n}$ for some sequence $a_{n} \rightarrow \infty$, this paper gives asymptotic expressions for $P\left(Y_{n} \geq y_{n}\right)$, which we call strong large deviation results. These results are found in Theorems 3.3 and 3.5 which impose conditions on the moment generating function (m.g.f.) of $T_{n}$. These extend the well-known strong large deviation results for sums of i.i.d. random variables due to Bahadur and Ranga Rao (1960).

The proofs of our strong large deviation theorems depend on the local limit results for $Y_{n}$. These are established in this paper in Theorems 2.1, 2.2, 2.3 and 2.9 and they are in the spirit of Feller (1967) wherein can be found some of the first local limit results for sums of i.i.d. random variables. Local limit results for extreme values are established in de Haan and Resnick (1982). Local limit results for sums of triangular arrays of i.i.d. random variables can be found in Jain and Pruitt (1987). The local limit results in this paper apply to arbitrary random variables $Y_{n}$ and require some easily verifiable boundedness conditions on their characteristic functions.

We illustrate our general results with two applications in Section 4. The first application is a local limit result for sums of dependent random variables given by a general model considered in Chaganty and Sethuraman (1987). The second application is a strong large deviation result for the Wilcoxon signedrank statistic under the null hypothesis.

We do not study large deviation local limit results in this paper. We have obtained such results for arbitrary random variables in Chaganty and Sethuraman (1985) for one-dimensional random variables and in Chaganty and Sethuraman (1986) for multidimensional random variables.

2. Local limit theorems. Let $\left\{Y_{n}, n \geq 1\right\}$ be a sequence of real valued random variables which converge weakly to a random variable $Y$. Then $\hat{f}_{n}(t) \rightarrow \hat{f}(t)$ for each $t$, where $\hat{f}_{n}(t)$ and $\hat{f}(t)$ are the characteristic functions (c.f.) of $Y_{n}$ and $Y$, respectively. In this section we show that if $\hat{f}_{n}(t)$ satisfies some boundedness conditions, then the p.d.f. of $Y_{n}$, or more generally the pseudodensity function of $Y_{n}$ converges uniformly to the p.d.f. of $Y$. Both the cases where $Y_{n}$ is nonlattice valued and lattice valued will be considered. To motivate the boundedness conditions used in the main Theorems 2.3 and 2.9 of this section, we begin with two theorems, using bounds on $\hat{f}_{n}(t)$ over the whole real line, which are straightforward and must be well known.

THEOREM 2.1. Let $\left\{Y_{n}, n \geq 1\right\}$ be a sequence of real valued random variables which converge weakly to a random variable $Y$. Let $\hat{f}_{n}(t)$ and $\hat{f}(t)$ be the c.f.'s of $Y_{n}$ and $Y$, respectively. Suppose that there exists an integrable function 
$f^{*}(t)$ such that

$$
\sup _{n}\left|\hat{f}_{n}(t)\right| \leq f^{*}(t)
$$

for all $t$. Then $Y_{n}$ possesses a bounded and continuous p.d.f. $f_{n}, Y$ also possesses a bounded and continuous p.d.f. $f$, and $f_{n}\left(y_{n}\right)$ converges to $f\left(y^{*}\right)$ if $y_{n} \rightarrow y^{*}$.

Proof. Condition (2.1) implies that the c.f.'s $\hat{f}_{n}$ and $\hat{f}$ are integrable. Hence both $Y_{n}$ and $Y$ possess bounded and continuous p.d.f.'s. The inversion formula and the dominated convergence theorem show that $f_{n}\left(y_{n}\right)$ converges to $f\left(y^{*}\right)$ if $y_{n} \rightarrow y^{*}$ as $n \rightarrow \infty$.

A random variable $X$ is said to be lattice valued with span $p$ and displacement $c$ if $P(X \in L)=1$, where $L=\{c+k p$, for some $k=$ $0, \pm 1, \pm 2, \ldots\}$ and $0 \leq c<p$ and $p$ is the largest such number. Some authors say that $X$ is lattice valued only when $c=0$; in this paper we do not make this distinction. A lattice valued random variable cannot satisfy condition (2.1) since the modulus of its c.f. is periodic. Hence Theorem 2.1 is not applicable to lattice valued random variables. The following theorem will apply to lattice valued random variables.

THEOREM 2.2. Let $Y_{n}$ be lattice valued random variables with span $h_{n}$ converging to 0 . Let $Y_{n}$ converge weakly to $Y$. Assume that there exists an integrable function $f^{*}$ such that

$$
\sup _{n}\left|\hat{f}_{n}(t)\right| I\left(|t| \leq \pi / h_{n}\right) \leq f^{*}(t)
$$

for each $t$. Then $Y$ possesses a bounded and continuous p.d.f. f, and there exists a constant $M<\infty$ such that

$$
\sup _{n} \sup _{y}\left[\frac{1}{h_{n}} P\left(Y_{n}=y\right)\right] \leq M
$$

Further, if $y_{n}$ is in the range of $Y_{n}$, and $y_{n}$ converges to $y^{*}$ then

$$
\frac{1}{h_{n}} P\left(Y_{n}=y_{n}\right) \rightarrow f\left(y^{*}\right)
$$

as $n \rightarrow \infty$.

Proof. Let $y_{n}$ be in the range of $Y_{n}$. Then an application of the inversion formula yields

$$
\frac{1}{h_{n}} P\left(Y_{n}=y_{n}\right)=\frac{1}{2 \pi} \int_{-\pi / h_{n}}^{\pi / h_{n}} \exp \left(-i t y_{n}\right) \hat{f}_{n}(t) d t
$$


The assertions (2.3) and (2.4) now follow from condition (2.2) and the dominated convergence theorem.

The conditions (2.1) in the nonlattice case and (2.2) in the lattice case are too strong to be useful in most situations. We show in Theorems 2.3 and 2.9 that appropriate bounds on the c.f. $\hat{f}_{n}(t)$ on increasing sequences of bounded intervals are sufficient to obtain results similar to those of Theorems 2.1 and 2.2 .

Since $Y_{n}$ may not possess a p.d.f., we will define its pseudodensity function at $y$ as the p.d.f. of $Y_{n}+Z_{n}$, where $Z_{n}$ is independent of $Y_{n}$ and uniformly distributed on $-S / b_{n}$, where $b_{n} \rightarrow \infty$ and $S$ is a set such that $0<\mu\left(S^{0}\right)=$ $\mu(\bar{S})<\infty$, where $\mu$ is the Lebesgue measure on $R$. More directly, the pseudodensity $q_{n}\left(y ; b_{n}, S\right)$ of $Y_{n}$ is defined by

$$
q_{n}\left(y ; b_{n}, S\right)=\frac{b_{n}}{\mu(S)} P\left(b_{n}\left(Y_{n}-y\right) \in S\right) .
$$

We will keep the set $S$ fixed throughout this section when dealing with pseudodensities. Let $\left\{y_{n}\right\}$ be a sequence of real numbers such that $y_{n} \rightarrow y^{*}$. The convergence of $q_{n}\left(y_{n} ; b_{n}, S\right)$ to the p.d.f. of $Y$ at $y^{*}$ will be referred to as a local limit theorem in this paper. This is the spirit under which local limit theorems have been studied for normalized sums of i.i.d. random variables by Feller (1967), for normalized extreme values in de Haan and Resnick (1982) and for normalized triangular arrays of i.i.d. random variables in Jain and Pruitt (1987).

THEOREM 2.3. Let $\left\{Y_{n}, n \geq 1\right\}$ be a sequence of real valued random variables which converge weakly to a random variable $Y$. Let $\hat{f}_{n}(t)$ and $\hat{f}(t)$ be the c. $f$.' $s$ of $Y_{n}$ and $Y$, respectively. Suppose that there exist an integrable function $f^{*}(t)$ and sequences $\left\{\beta_{n}\right\}$ and $\left\{b_{n}\right\}$ with $\beta_{n} \rightarrow \infty, b_{n} \rightarrow \infty$, such that

$$
\sup _{n}\left|\hat{f}_{n}(t)\right| I\left(|t| \leq \beta_{n}\right) \leq f^{*}(t)
$$

for each $t$, and

$$
\theta_{n}(\lambda)=\sup _{\beta_{n}<|t| \leq \lambda b_{n}}\left|\hat{f}_{n}(t)\right|=o\left(\frac{1}{b_{n}}\right)
$$

for each $\lambda>0$, where the above supremum is defined to be 0 if $\left\{t: \beta_{n}<|t| \leq\right.$ $\left.\lambda b_{n}\right\}$ is empty. Then the random variable $Y$ possesses a bounded and continuous p.d.f. f. Let $q_{n}\left(y ; b_{n}, S\right)$ be the pseudodensity function of $Y_{n}$ as defined in (2.6). Then there exists a finite constant $M$ and an integer $n_{s}$, which may depend on $S$, such that

$$
\sup _{y}\left[q_{n}\left(y ; b_{n}, S\right)\right] \leq M
$$


for $n \geq n_{s}$. Furthermore, if $y_{n} \rightarrow y^{*}$, then

$$
q_{n}\left(y_{n} ; b_{n}, S\right) \rightarrow f\left(y^{*}\right)
$$

as $n \rightarrow \infty$.

Proof. Since $\hat{f}_{n}(t) \rightarrow \hat{f}(t)$ pointwise and $\beta_{n} \rightarrow \infty$, condition (2.7) implies that $\hat{f}$ is bounded by $f^{*}$. Hence $Y$ possesses a bounded and continuous p.d.f. $f$. Suppose $\beta_{n} / b_{n}$ is bounded. Since $b_{n} \theta_{n}(\lambda) \rightarrow 0$, for each $\lambda>0$, we can find a sequence $\left\{\lambda_{n}\right\}$ satisfying

$$
\lambda_{n} \rightarrow \infty \text { and } \lambda_{n} b_{n} \theta_{n} \rightarrow 0
$$

as $n \rightarrow \infty$, where $\theta_{n}={ }_{\text {def }} \theta_{n}\left(\lambda_{n}\right)$. Now, suppose that $\beta_{n} / b_{n} \rightarrow \infty$. In this case we let $\lambda_{n}=\beta_{n} / b_{n}$ and (2.11) is satisfied because $\theta_{n}={ }_{\text {def }} \theta_{n}\left(\lambda_{n}\right)=0$. Let $U_{n}$ be the uniform distribution on the set $-S / b_{n}$ and $u_{n}, \hat{u}_{n}$ be the p.d.f. and c.f. corresponding to $U_{n}$. We also introduce another distribution function (d.f.) $V_{n}$ with p.d.f. $v_{n}$ and c.f. $\hat{v}_{n}$ which vanishes outside of $\left[-\lambda_{n} b_{n}, \lambda_{n} b_{n}\right]$, to obtain the important identity (2.14):

$$
\begin{aligned}
& \left.v_{n}(x)=\frac{\lambda_{n} b_{n}\left[\frac{\sin \left(\lambda_{n} b_{n} x / 2\right)}{2 \pi}\right]^{2}, \quad-\infty<x<\infty,}{\left(\lambda_{n} b_{n} x / 2\right)}\right] \\
& \hat{v}_{n}(t)= \begin{cases}1-\frac{|t|}{\lambda_{n} b_{n}}, & \text { if }|t| \leq \lambda_{n} b_{n}, \\
0, & \text { otherwise. }\end{cases}
\end{aligned}
$$

Let $F_{n}$ be the d.f. of $Y_{n}$, and let $Q_{n}=F_{n} * U_{n}, M_{n}=Q_{n} * V_{n}$, where $*$ denotes the convolution operation. Notice that $q_{n}\left(y ; b_{n}, S\right)$ defined in (2.6) is the p.d.f. of $Q_{n}$. Let $m_{n}(y)$ be the p.d.f. of $M_{n}$. The c.f. $\hat{m}_{n}(t)$ of $M_{n}$, which is equal to $\hat{f}_{n}(t) \hat{u}_{n}(t) \hat{v}_{n}(t)$, vanishes outside the interval $\left[-\lambda_{n} b_{n}, \lambda_{n} b_{n}\right]$. The inversion theorem yields the following identity:

$$
\begin{aligned}
m_{n}(y) & =\int_{-\infty}^{\infty} q_{n}\left(y-x ; b_{n}, S\right) v_{n}(x) d x \\
& =\frac{b_{n}}{\mu(S)} \int_{-\infty}^{\infty} P\left(b_{n}\left(Y_{n}-y+x\right) \in S\right) v_{n}(x) d x \\
& =\frac{1}{2 \pi} \int_{-\lambda_{n} b_{n}}^{\lambda_{n} b_{n}} \exp (-i t y) \hat{m}_{n}(t) d t
\end{aligned}
$$

Relation (2.14) is the starting point of the main part of this proof and it relates $q_{n}\left(y ; b_{n}, S\right)$ to the integrable c.f. $\hat{m}_{n}(t)$. We first show that $m_{n}\left(y_{n}\right)$ converges to $f\left(y^{*}\right)$ and then obtain lower and upper bounds for $m_{n}\left(y_{n}\right)$ which depend on $q_{n}\left(y_{n} ; b_{n}, S\right)$. This will then establish (2.9) and (2.10). Notice that from (2.11) we get

$$
\left|\frac{1}{2 \pi} \int_{\beta_{n}<|t| \leq \lambda_{n} b_{n}} \exp \left(-i t y_{n}\right) \hat{m}_{n}(t) d t\right| \leq \frac{\lambda_{n} b_{n} \theta_{n}}{\pi} \rightarrow 0 .
$$


From condition (2.7), the dominated convergence theorem and the inversion formula we get

$$
\frac{1}{2 \pi} \int_{-\beta_{n}}^{\beta_{n}} \exp \left(-i t y_{n}\right) \hat{m}_{n}(t) d t \rightarrow \frac{1}{2 \pi} \int_{-\infty}^{\infty} \exp \left(-i t y^{*}\right) \hat{f}(t) d t=f\left(y^{*}\right)
$$

This shows that

$$
m_{n}\left(y_{n}\right) \rightarrow f\left(y^{*}\right)
$$

Let $\eta>0$. Let $s(x, \eta)$ be a closed interval centered at $x$, that is, $s(x, \eta)=$ $\{y:|y-x| \leq \eta\}$. Let $S_{\eta}=\{x: s(x, \eta) \subset S\}$ and $S^{\eta}=\{y:|y-x| \leq \eta$, for some $x \in S\}$. Since we have assumed that $\mu\left(S^{0}\right)=\mu(\bar{S})$ we can find $\eta\left(=\eta_{s}\right)>0$ such that

$$
\mu\left(S_{\eta}\right)>0 \text { and }\left[\mu\left(S^{\eta}\right) / \mu(S)\right] \leq 2 .
$$

Note that $y \in S_{\eta}$ implies that $y+x \in s(y, \eta) \subset S$ if $|x| \leq \eta$. From this, we get a lower bound for $m_{n}(y)$ as follows:

$$
\begin{aligned}
m_{n}(y) & \geq \frac{b_{n}}{\mu(S)} \int_{|x| \leq \eta / b_{n}} P\left(b_{n}\left(Y_{n}-y+x\right) \in S\right) v_{n}(x) d x \\
& \geq \frac{b_{n}}{\mu(S)} P\left(b_{n}\left(Y_{n}-y\right) \in S_{\eta}\right) \int_{|x| \leq \eta / b_{n}} v_{n}(x) d x \\
& \geq \frac{b_{n}}{\mu(S)} P\left(b_{n}\left(Y_{n}-y\right) \in S_{\eta}\right)\left[1-\frac{4}{\pi \lambda_{n} \eta}\right] .
\end{aligned}
$$

Using (2.14), (2.15), (2.19) and condition (2.7) we get

$$
\begin{aligned}
\frac{b_{n}}{\mu(S)} & P\left(b_{n}\left(Y_{n}-y\right) \in S_{\eta}\right)\left[1-\frac{4}{\pi \lambda_{n} \eta}\right] \\
& \leq m_{n}(y) \leq \frac{\lambda_{n} b_{n} \theta_{n}}{\pi}+\frac{1}{2 \pi} \int_{-\infty}^{\infty} f^{*}(t) d t \\
& \leq \frac{1}{\pi} \int_{-\infty}^{\infty} f^{*}(t) d t
\end{aligned}
$$

for sufficiently large $n$. By replacing $S$ by $S^{\eta}$ and using (2.18) and the fact that $S \subset\left(S^{\eta}\right)_{\eta}$, we get

$$
\begin{aligned}
& \frac{b_{n}}{\mu(S)} P\left(b_{n}\left(Y_{n}-y\right) \in S\right)\left[1-\frac{4}{\pi \lambda_{n} \eta}\right] \\
& \quad \leq \frac{\mu\left(S^{\eta}\right)}{\mu(S)} \frac{1}{\pi} \int_{-\infty}^{\infty} f^{*}(t) d t \leq \frac{2}{\pi} \int_{-\infty}^{\infty} f^{*}(t) d t .
\end{aligned}
$$

Since $\lambda_{n} \rightarrow \infty$ as $n \rightarrow \infty$ we can find an integer $n_{s}$ so that

$$
\sup _{y}\left[\frac{b_{n}}{\mu(S)} P\left(b_{n}\left(Y_{n}-y\right) \in S\right)\right] \leq M
$$


for $n \geq n_{s}$, where

$$
M=\frac{3}{\pi} \int_{-\infty}^{\infty} f^{*}(t) d t .
$$

This proves assertion (2.9). Note that $y \in S$ implies that $y-x \in S^{\eta}$ for $|x| \leq \eta$. Therefore for $n \geq n_{s}$ an upper bound for $m_{n}(y)$ is given by

$$
\begin{aligned}
m_{n}(y)= & \frac{b_{n}}{\mu(S)} \int_{-\infty}^{\infty} P\left(b_{n}\left(Y_{n}-y+x\right) \in S\right) v_{n}(x) d x \\
\leq & \frac{b_{n}}{\mu(S)} P\left(b_{n}\left(Y_{n}-y\right) \in S^{\eta}\right) \int_{|x| \leq \eta / b_{n}} v_{n}(x) d x \\
& +M \int_{|x|>\eta / b_{n}} v_{n}(x) d x \\
\leq & \frac{b_{n}}{\mu(S)} P\left(b_{n}\left(Y_{n}-y\right) \in S^{\eta}\right)+\frac{4 M}{\pi \lambda_{n} \eta} .
\end{aligned}
$$

Thus, from (2.17), (2.19) and (2.24) we get that

$$
\begin{aligned}
& \underset{n}{\limsup } \frac{b_{n}}{\mu(S)} P\left(b_{n}\left(Y_{n}-y_{n}\right) \in S_{\eta}\right) \\
& \quad \leq f\left(y^{*}\right) \leq \liminf _{n} \frac{b_{n}}{\mu(S)} P\left(b_{n}\left(Y_{n}-y_{n}\right) \in S^{\eta}\right) .
\end{aligned}
$$

By replacing $S$ by $S^{\eta}$ in the 1.h.s. and $S$ by $S_{\eta}$ in the r.h.s. and using the relations $S \subset\left(S^{\eta}\right)_{\eta}$ and $\left(S_{\eta}\right)^{\eta} \subset S$ we get that

$$
\begin{aligned}
& \limsup _{n} \frac{b_{n}}{\mu\left(S^{\eta}\right)} P\left(b_{n}\left(Y_{n}-y_{n}\right) \in S\right) \\
& \quad \leq f\left(y^{*}\right) \leq \liminf _{n} \frac{b_{n}}{\mu\left(S_{\eta}\right)} P\left(b_{n}\left(Y_{n}-y_{n}\right) \in S\right) .
\end{aligned}
$$

Letting $\eta \rightarrow 0$ and using the fact $\mu\left(S^{0}\right)=\mu(\bar{S})$ we get the assertion (2.10).

COROLLARY 2.4. Suppose that the c.f.'s of the sequence $Y_{n}$ satisfy condition (2.1), which is stronger than condition (2.7). Then (2.9) and (2.10) hold for any sequence $b_{n} \rightarrow \infty$.

REMARK 2.5. The conclusions of Theorem 2.3 hold if we replace condition (2.8) by

$$
\int_{\beta_{n}<|t| \leq \lambda b_{n}}\left|\hat{f}_{n}(t)\right| d t \rightarrow 0 \quad \text { as } n \rightarrow \infty,
$$

for each $\lambda>0$. Notice that condition (2.8) is needed to obtain a sequence $\left\{\lambda_{n}\right\}$ satisfying (2.11) and to show that $m_{n}\left(y_{n}\right) \rightarrow f\left(y^{*}\right)$. If (2.27) holds for each 
$\lambda>0$, we can find a sequence of real numbers $\left\{\lambda_{n}\right\}$, such that $\lambda_{n} \rightarrow \infty$ and

$$
\int_{\beta_{n}<|t| \leq \lambda_{n} b_{n}}\left|\hat{f}_{n}(t)\right| d t \rightarrow 0 \text { as } n \rightarrow \infty .
$$

These are enough to show that $m_{n}\left(y_{n}\right) \rightarrow f\left(y^{*}\right)$, following the proof of Theorem 2.3.

The next theorem provides a convenient way to verify condition (2.7) of Theorem 2.3. In Lemma 3.3 of Section 3 we will use Theorem 2.3 and this method of verification of condition (2.7).

THEOREM 2.6. Let $\left\{Y_{n}, n \geq 1\right\}$ be a sequence of random variables with c.f.'s $\left\{\hat{f}_{n}(t)\right\}$. Let $\left\{d_{n}\right\}$ be a sequence of real numbers such that $d_{n} \rightarrow \infty$. Assume that there exists $\delta>0$ such that $g_{n}(t)=d_{n}^{-2} \log \left|\hat{f}_{n}\left(d_{n} t\right)\right|$ is finite and twice differentiable in the interval $[-\delta, \delta]$, for all $n \geq 1$. Suppose that there exists $\alpha>0$ such that for $|t| \leq \delta$,

$$
-g_{n}^{\prime \prime}(t) \geq \alpha
$$

for all $n \geq 1$. Then condition (2.7) of Theorem 2.3 is satisfied with $\beta_{n}=\delta d_{n}$.

Proof. By expanding $g_{n}(t)$ around 0 by Taylor's theorem, we find that, for $|t| \leq \delta$,

$$
\begin{aligned}
g_{n}(t) & =g_{n}(0)+\operatorname{tg}_{n}^{\prime}(0)+\frac{t^{2}}{2} g_{n}^{\prime \prime}\left(r_{n}\right) \\
& =\frac{t^{2}}{2} g_{n}^{\prime \prime}\left(r_{n}\right) \\
& \leq-\frac{\alpha t^{2}}{2},
\end{aligned}
$$

where $r_{n}$ is such that $\left|r_{n}\right|<|t| \leq \delta$. Let $\beta_{n}=\delta d_{n}$. Thus for $|t| \leq \beta_{n}$, we have for all $n \geq 1$,

$$
\begin{aligned}
\left|\hat{f}_{n}(t)\right| & =\exp \left(d_{n}^{2}\left(g_{n}\left(t / d_{n}\right)\right)\right) \\
& \leq \exp \left(-\alpha t^{2} / 2\right),
\end{aligned}
$$

which is an integrable function. This completes the proof of the theorem.

The next theorem obtains the limit of a function related to the Laplace transform of the positive part of $Y_{n}$ when (2.9) and (2.10) hold. It plays an important role in the proofs of the strong large deviation theorems of Section 3.

THEOREM 2.7. Let $\left\{Y_{n}, n \geq 1\right\}$ be a sequence of random variables converging weakly to $Y$, which possess a p.d.f. f. Let $\left\{b_{n}\right\}$ be a sequence of real numbers such that $b_{n} \rightarrow \infty$. Let $q_{n}\left(y ; b_{n}, S\right)$ be as defined in (2.6). Assume that 
$q_{n}\left(y ; b_{n}, S\right)$ satisfies (2.9) and (2.10). Then

$$
b_{n} E\left[\exp \left(-b_{n} Y_{n}\right) I\left(Y_{n} \geq 0\right)\right] \rightarrow f(0)
$$

as $n \rightarrow \infty$.

Proof. Let $h>0$. Consider

$$
\begin{aligned}
I_{n} & =E\left[\exp \left(-b_{n} Y_{n}\right) I\left(Y_{n} \geq 0\right)\right] \\
& =\sum_{k=1}^{\infty} E\left[\exp \left(-b_{n} Y_{n}\right) I\left(\frac{(k-1) h}{b_{n}} \leq Y_{n}<\frac{k h}{b_{n}}\right)\right] \\
& =\sum_{k=1}^{\infty} E\left[\exp \left(-b_{n} Y_{n}\right) I\left(-\frac{h}{2 b_{n}} \leq Y_{n}-y_{n k}<\frac{h}{2 b_{n}}\right)\right],
\end{aligned}
$$

where $y_{n k}=(2 k-1) h / 2 b_{n}$. Let $k_{h}=\left[1 / h^{2}\right]$ and $S_{h}=[-h / 2, h / 2)$. We now get lower and upper bounds for $I_{n}$ as follows:

$$
\begin{aligned}
I_{n} & \geq \sum_{k=1}^{k_{h}} \exp (-k h) P\left(-\frac{h}{2 b_{n}} \leq Y_{n}-y_{n k}<\frac{h}{2 b_{n}}\right) \\
& =\frac{h}{b_{n}} \sum_{k=1}^{k_{h}} \exp (-k h) q_{n}\left(y_{n k} ; b_{n}, S_{h}\right)
\end{aligned}
$$

and

$$
\begin{aligned}
I_{n} \leq & \sum_{k=1}^{\infty} \exp (-(k-1) h) P\left(-\frac{h}{2 b_{n}} \leq Y_{n}-y_{n k}<\frac{h}{2 b_{n}}\right) \\
= & \frac{h}{b_{n}} \sum_{k=1}^{k_{h}} \exp (-(k-1) h) q_{n}\left(y_{n k} ; b_{n}, S_{h}\right) \\
& +\frac{h}{b_{n}} \sum_{k=k_{h}+1}^{\infty} \exp (-(k-1) h) q_{n}\left(y_{n k} ; b_{n}, S_{h}\right) .
\end{aligned}
$$

Using (2.9) and (2.10) after noting that $y_{n k} \rightarrow 0$ as $n \rightarrow \infty$ for each $k$, we get

$$
\begin{aligned}
\liminf _{n}\left(b_{n} I_{n}\right) & \geq f(0) h \sum_{k=1}^{k_{h}} \exp (-k h) \\
& =f(0) \frac{h\left(\exp (-h)-\exp \left(-\left(k_{h}+1\right) h\right)\right)}{1-\exp (-h)}
\end{aligned}
$$

and

$$
\begin{aligned}
\underset{n}{\limsup \left(b_{n} I_{n}\right) \leq} & f(0) h \sum_{k=1}^{k_{h}} \exp (-(k-1) h) \\
& +M h \sum_{k=k_{h}+1}^{\infty} \exp (-(k-1) h) \\
= & f(0) \frac{h\left(1-\exp \left(-k_{h} h\right)\right)}{1-\exp (-h)}+M h \frac{\exp \left(-k_{h} h\right)}{1-\exp (-h)}
\end{aligned}
$$


Letting $h \rightarrow 0$ in (2.36) and (2.37) we get

$$
\lim _{n}\left(b_{n} I_{n}\right)=f(0) \text {. }
$$

This completes the proof of the lemma.

REMARK 2.8. The conditions used in Theorem 2.3 can be satisfied by both nonlattice and lattice random variables. For a lattice valued random variable $Y_{n}$ with span $h_{n}$, condition (2.7) can be satisfied only if $h_{n}$ converges to 0 and condition (2.8) can be satisfied only if $b_{n} h_{n}$ converges to 0 . We will now extend Theorems 2.3 and 2.7 to the case where $0<\liminf _{n} b_{n} h_{n}<\infty$. Thus we might as well take $b_{n}=1 / h_{n}$. We can also take $S=(-1 / 2,1 / 2)$ and notice that

$$
q_{n}\left(y ; b_{n}, S\right)=\frac{1}{h_{n}} P\left(Y_{n}=y\right)
$$

where $y$ is in the range of $Y_{n}$.

THEOREM 2.9. Let $\left\{Y_{n}, n \geq 1\right\}$ be lattice valued random variables with span $h_{n}$ converging to 0 . Let $Y_{n}$ converge weakly to $Y$. Let $\hat{f}_{n}(t)$ and $\hat{f}(t)$ be the c.f.'s of $Y_{n}$ and $Y$, respectively. Assume that there exists an integrable function $f^{*}$ such that

$$
\sup _{n}\left|\hat{f}_{n}(t)\right| I\left(|t| \leq \beta_{n}\right) \leq f^{*}(t)
$$

for each $t$, and

$$
\theta_{n}^{*}=\sup _{\beta_{n}<|t| \leq \pi / h_{n}}\left|\hat{f}_{n}(t)\right|=o\left(h_{n}\right),
$$

for some sequence of real numbers $\left\{\beta_{n}\right\}$ such that $\beta_{n} \rightarrow \infty$ and $\beta_{n}<\pi / h_{n}$ for all $n \geq 1$. Then $Y$ possesses a bounded and continuous p.d.f. $f$, and there exists a constant $M$ such that (2.3) holds. If $y_{n}$ is in the range of $Y_{n}$ and $y_{n}$ converges to $y^{*}$ as $n \rightarrow \infty$, then (2.4) holds.

Proof. Let $y_{n}$ be a possible value of $Y_{n}$. Then an application of the inversion formula yields

$$
\begin{aligned}
\frac{1}{h_{n}} P\left(Y_{n}=y_{n}\right)= & \frac{1}{2 \pi} \int_{-\pi / h_{n}}^{\pi / h_{n}} \exp \left(-i t y_{n}\right) \hat{f}_{n}(t) d t \\
= & \frac{1}{2 \pi} \int_{|t| \leq \beta_{n}} \exp \left(-i t y_{n}\right) \hat{f}_{n}(t) d t \\
& +\frac{1}{2 \pi} \int_{\beta_{n}<|t| \leq \pi / h_{n}} \exp \left(-i t y_{n}\right) \hat{f}_{n}(t) d t \\
= & I_{n 1}+I_{n 2}, \text { say. }
\end{aligned}
$$

It is easy to check that condition (2.40) and the dominated convergence 
theorem imply that $I_{n 1}$ converges to $(1 / 2 \pi) \int \exp \left(-i t y^{*}\right) \hat{f}(t) d t=f\left(y^{*}\right)$. Next,

$$
\begin{aligned}
\left|I_{n 2}\right| & \leq \frac{1}{h_{n}} \sup _{\beta_{n}<|t| \leq \pi / h_{n}}\left|\hat{f}_{n}(t)\right| \\
& =\frac{\theta_{n}^{*}}{h_{n}},
\end{aligned}
$$

which converges to 0 by condition (2.41), as $n \rightarrow \infty$. This completes the proof of (2.4). Next, from (2.42) and (2.43) we get

$$
\begin{aligned}
\sup _{y}\left[\frac{1}{h_{n}} P\left(Y_{n}=y\right)\right] & \leq \frac{1}{2 \pi} \int_{|t| \leq \beta_{n}}\left|\hat{f}_{n}(t)\right| d t+\frac{\theta_{n}^{*}}{h_{n}} \\
& \leq \frac{1}{2 \pi} \int_{-\infty}^{\infty} f^{*}(t) d t+\frac{\theta_{n}^{*}}{h_{n}} \\
& \leq \frac{1}{\pi} \int_{-\infty}^{\infty} f^{*}(t) d t=M
\end{aligned}
$$

for $n \geq n_{0}$. Hence (2.3) holds. This completes the proof of the theorem.

Theorem 2.10. Let $Y_{n}$ be a lattice valued random variable taking values in the lattice $\left\{k h_{n}: k=0, \pm 1, \pm 2, \ldots\right\}$, where $h_{n}>0$ for $n \geq 1$. Assume that the span $h_{n}$ of $Y_{n}$ converges to 0 as $n \rightarrow \infty$. Let $Y_{n}$ converge in distribution to $Y$. Let $\left\{b_{n}\right\}$ be a sequence of real numbers such that $0<\lim _{n} \inf _{n}\left(b_{n} h_{n}\right)=b<\infty$. Suppose that $Y$ possesses a p.d.f. f and $Y_{n}$ satisfies the conditions (2.3) and (2.4). Then

$$
\frac{\left(1-\exp \left(-b_{n} h_{n}\right)\right)}{h_{n}} E\left[\exp \left(-b_{n} Y_{n}\right) I\left(Y_{n} \geq 0\right)\right] \rightarrow f(0)
$$

as $n \rightarrow \infty$.

Proof. Consider

$$
\begin{aligned}
I_{n} & =E\left(\exp \left(-b_{n} Y_{n}\right) I\left(Y_{n} \geq 0\right)\right) \\
& =\sum_{k=0}^{\infty} \exp \left(-k b_{n} h_{n}\right) P\left(Y_{n}=k h_{n}\right) .
\end{aligned}
$$

Let $N>1$ be fixed. A lower bound for $I_{n}$ is given by

$$
\sum_{k=0}^{N-1} \exp \left(-k b_{n} h_{n}\right) P\left(Y_{n}=k h_{n}\right)
$$

and an upper bound is given by

$$
\sum_{k=0}^{N-1} \exp \left(-k b_{n} h_{n}\right) P\left(Y_{n}=k h_{n}\right)+M h_{n} \sum_{k=N}^{\infty} \exp \left(-k b_{n} h_{n}\right)
$$

wherein we have used (2.3). Combining (2.46), (2.47), (2.48) and using (2.4) we 
get

$$
\begin{aligned}
\liminf _{n}\left[\frac{\left(1-\exp \left(-b_{n} h_{n}\right)\right)}{h_{n}} I_{n}\right] & \geq f(0) \liminf _{n}\left(1-\exp \left(-N b_{n} h_{n}\right)\right) \\
& =f(0)(1-\exp (-N b))
\end{aligned}
$$

and

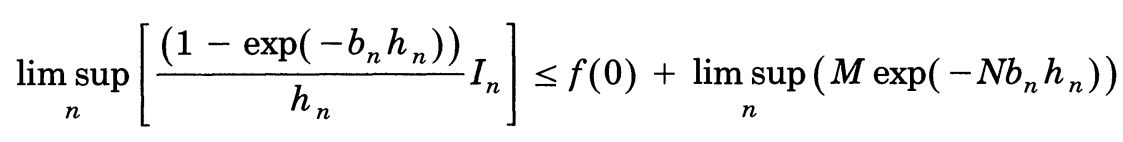

$$
\begin{aligned}
& =f(0)+M \exp (-N b),
\end{aligned}
$$

where $b=\liminf { }_{n}\left(b_{n} h_{n}\right)$. We let $N \rightarrow \infty$ in (2.49) and (2.50) and conclude that

$$
\lim _{n}\left[\frac{\left(1-\exp \left(-b_{n} h_{n}\right)\right)}{h_{n}} I_{n}\right]=f(0) .
$$

This completes the proof of the theorem.

3. Strong large deviation theorems. Let $\left\{T_{n}, n \geq 1\right\}$ be a sequence of random variables. Let $\left\{a_{n}\right\}$ be a sequence of real numbers and $\left\{m_{n}\right\}$ be a bounded sequence of real numbers. Weak large deviation results give asymptotic expressions for $\log P\left(T_{n} / a_{n} \geq m_{n}\right)$ where the event $\left\{T_{n} / a_{n} \geq m_{n}\right\}$ represents a large deviation. A number of authors, including Sievers (1969), Steinebach (1978) and Ellis (1984) have obtained such results under suitable conditions on the m.g.f. of $T_{n}$. Strong large deviation results give asymptotic expressions for $P\left(T_{n} / a_{n} \geq m_{n}\right)$. One of the earliest strong large deviation theorems was obtained by Bahadur and Ranga Rao (1960) when $T_{n}$ is the sum of i.i.d. random variables. In Theorems 3.3 and 3.5 of this section we obtain strong large deviation limit theorems for arbitrary sequences of random variables $\left\{T_{n}, n \geq 1\right\}$, under some conditions on the m.g.f.'s of $T_{n}$ 's. In Remark 3.6 we demonstrate that Theorems 3.3. and 3.5 are sufficient to establish the strong large deviation results for sums of i.i.d. random variables obtained by Bahadur and Ranga Rao (1960). This shows that the generalization to general random variables in Theorems 3.3 and 3.5 has not been obtained by introducing unnecessary or restrictive conditions. The proofs of our strong large deviation results depend heavily on the local limit theorems of Section 2 . We use the notation $A_{n} \sim B_{n}$, if $A_{n} / B_{n} \rightarrow 1$. We shall develop some more notation before stating the main theorem.

Let $\left\{T_{n}, n \geq 1\right\}$ be a sequence of random variables with m.g.f. $\phi_{n}(z)=$ $E\left[\exp \left(z T_{n}\right)\right]$, which is nonvanishing and analytic in the region $\Omega=\{z \in \mathscr{b}$ : $|z|<a\}$, where $a>0$ and $b$ is the set of all complex numbers. Let $\left\{a_{n}\right\}$ be a 
sequence of real numbers. Let

$$
\begin{aligned}
& \psi_{n}(z)=a_{n}^{-1} \log \phi_{n}(z), \quad \text { for } z \in \Omega, \\
& \gamma_{n}(u)=\sup _{|s|<a, s \in R_{1}}\left[u s-\psi_{n}(s)\right], \quad \text { for } u \in R_{1} .
\end{aligned}
$$

Note that $\psi_{n}$ is a convex function on $(-a, a)$. Let $\left\{m_{n}\right\}$ be a bounded sequence of real numbers such that there exists a sequence $\left\{\tau_{n}\right\}$ satisfying

$$
\psi_{n}^{\prime}\left(\tau_{n}\right)=m_{n} \text { and } 0<\tau_{n}<a_{0}<a, \text { for all } n \geq 1 \text {. }
$$

Under these conditions we can see that $\gamma_{n}\left(m_{n}\right)=m_{n} \tau_{n}-\psi_{n}\left(\tau_{n}\right)$. Let $K_{n}$ be the d.f. of $T_{n}$. We will use the left continuous version of the distribution function which will enable us to write the identities in (3.5). Let

$$
H_{n}(y)=\int_{-\infty<u<y} \exp \left(u \tau_{n}-a_{n} \psi_{n}\left(\tau_{n}\right)\right) d K_{n}(u)
$$

and let $T_{n}^{*}$ be a random variable with d.f. $H_{n}(y)$. Let $T_{n}^{\prime}=T_{n}^{*}-a_{n} m_{n}$, $Y_{n}=T_{n}^{\prime} / d_{n}, d_{n}=\sqrt{a_{n} \psi_{n}^{\prime \prime}\left(\tau_{n}\right)}$ and $b_{n}=\tau_{n} d_{n}$. Note that if $T_{n}$ is a lattice valued random variable with span $p_{n}>0$, we can assume, without loss of generality, when considering $P\left(T_{n} / a_{n} \geq m_{n}\right)$, that $m_{n}$ is in the range of $T_{n} / a_{n}$. In this case we can easily verify that $Y_{n}$ is a lattice valued random variable with span $h_{n}=p_{n} / d_{n}$ and displacement 0 . The study of the strong large deviation properties of $T_{n}$ is carried out by relating them to local limit results for the random variables $Y_{n}$ previously defined and using the identity (3.5) which follows. This identity is easily verified:

$$
\begin{aligned}
P\left(\frac{T_{n}}{a_{n}} \geq m_{n}\right) & =\int_{a_{n} m_{n}}^{\infty} d K_{n}(y) \\
& =\int_{a_{n} m_{n}}^{\infty} \exp \left(-y \tau_{n}+a_{n} \psi_{n}\left(\tau_{n}\right)\right) d H_{n}(y) \\
& =\exp \left(a_{n} \psi_{n}\left(\tau_{n}\right)\right) E\left(\exp \left(-\tau_{n} T_{n}^{*}\right) I\left(T_{n}^{*} \geq a_{n} m_{n}\right)\right) \\
& =\exp \left(-a_{n} \gamma_{n}\left(m_{n}\right)\right) E\left(\exp \left(-\tau_{n} T_{n}^{\prime}\right) I\left(T_{n}^{\prime} \geq 0\right)\right) \\
& =\exp \left(-a_{n} \gamma_{n}\left(m_{n}\right)\right) E\left(\exp \left(-b_{n} Y_{n}\right) I\left(Y_{n} \geq 0\right)\right) .
\end{aligned}
$$

This is usually called the use of the Esscher transformation, and is the starting point of most investigations in large deviations.

LEMma 3.1. Let $\left\{T_{n}, n \geq 1\right\}$ be a sequence of random variables. Let $\left\{m_{n}\right\}$ be a bounded sequence of real numbers such that there exists a sequence $\left\{\tau_{n}\right\}$ satisfying (3.3). Let $d_{n}=\sqrt{a_{n} \psi_{n}^{\prime \prime}\left(\tau_{n}\right)}$ and let $a_{n} \rightarrow \infty$. Let the random variable $Y_{n}$ be as defined following identity (3.4). Let $\hat{f}_{n}(t)$ be the c.f. of $Y_{n}$. Assume the following conditions for $T_{n}$ :

(a) There exists $\beta<\infty$ such that $\left|\psi_{n}(z)\right|<\beta$ for all $n \geq 1, z \in \Omega$.

(b) There exists $\alpha>0$ such that $\psi_{n}^{\prime \prime}\left(\tau_{n}\right) \geq \alpha$ for all $n \geq 1$. 
Then $Y_{n}$ converges in distribution to the standard normal and there exists $\delta>0$ such that

$$
\sup _{n}\left|\hat{f}_{n}(t)\right| I\left(|t| \leq \delta d_{n}\right) \leq \exp \left(-\alpha t^{2} / 2\right)
$$

Proof. The c.f. of $Y_{n}$ is given by

$$
\hat{f}(t)=\exp \left(-\frac{i t a_{n} m_{n}}{d_{n}}\right) \frac{\phi_{n}\left(\tau_{n}+i t / d_{n}\right)}{\phi_{n}\left(\tau_{n}\right)} .
$$

Since $\psi_{n}(z)=a_{n}^{-1} \log \phi_{n}(z)$ is a finite and analytic function in $\Omega$, and $0<\tau_{n}<$ $a_{0}$, using a Taylor series expansion, we can write, for $|t|<\left(a-a_{0}\right) / 2$,

(3.8) $\psi_{n}\left(\tau_{n}+i t\right)=\psi_{n}\left(\tau_{n}\right)+i t \psi_{n}^{\prime}\left(\tau_{n}\right)-\left(t^{2} / 2\right) \psi_{n}^{\prime \prime}\left(\tau_{n}\right)+R_{n}\left(\tau_{n}+i t\right)$, where the remainder term $R_{n}$ satisfies

$$
\left|R_{n}\left(\tau_{n}+i t\right)\right| \leq \frac{\beta|t|^{3}}{\left(\left(a-a_{0}\right) / 2\right)^{3}},
$$

from condition (a) and Cauchy's inequality [see Apostol (1974), page 451]. From (3.7), (3.8), (3.9) and condition (b) we obtain, for any fixed $t$, that

$$
\begin{aligned}
\log \hat{f}_{n}(t)= & -\left(i t a_{n} m_{n}\right) / d_{n}+a_{n}\left[\psi_{n}\left(\tau_{n}+i t / d_{n}\right)-\psi_{n}\left(\tau_{n}\right)\right] \\
= & -\left(i t a_{n} m_{n}\right) / d_{n}+a_{n}\left[i t \psi_{n}^{\prime}\left(\tau_{n}\right) / d_{n}\right. \\
\left.3.10) \quad-\left(t^{2} \psi_{n}^{\prime \prime}\left(\tau_{n}\right)\right) /\left(2 d_{n}^{2}\right)+R_{n}\left(\tau_{n}+i t / d_{n}\right)\right] & \\
= & -t^{2} / 2+a_{n} R_{n}\left(\tau_{n}+i t / d_{n}\right)
\end{aligned}
$$

and

(3.11) $\left|a_{n} R_{n}\left(\tau_{n}+i t / d_{n}\right)\right| \leq \frac{\beta|t|^{3}}{\alpha d_{n}\left(\left(a-a_{0}\right) / 2\right)^{3}} \rightarrow 0$, as $n \rightarrow \infty$.

Hence $Y_{n}$ converges weakly to the standard normal random variable. The conclusion (3.6) will follow from Theorem 2.6, if we verify that $Y_{n}$ satisfies condition (2.29). Let

$$
\begin{aligned}
g_{n}(t) & =d_{n}^{-2} \log \left|\hat{f}_{n}\left(d_{n} t\right)\right| \\
& =\frac{1}{\psi_{n}^{\prime \prime}\left(\tau_{n}\right)}\left[\operatorname{Real}\left(\psi_{n}\left(\tau_{n}+i t\right)-\psi_{n}\left(\tau_{n}\right)\right)\right] .
\end{aligned}
$$

Thus

$$
\begin{aligned}
g_{n}^{\prime \prime}(t) & =\frac{-\operatorname{Real}\left(\psi_{n}^{\prime \prime}\left(\tau_{n}+i t\right)\right)}{\psi_{n}^{\prime \prime}\left(\tau_{n}\right)} \\
& =\frac{-\operatorname{Real}\left(\psi_{n}^{\prime \prime}\left(\tau_{n}\right)+i t \xi_{n}\right)}{\psi_{n}^{\prime \prime}\left(\tau_{n}\right)} \\
& =-1-\operatorname{Real}\left(i t \xi_{n} / \psi_{n}^{\prime \prime}\left(\tau_{n}\right)\right) \\
& \leq-1+|t| \frac{\left|\xi_{n}\right|}{\alpha},
\end{aligned}
$$


where $\xi_{n}$ is an appropriate complex number depending on the third derivative of $\psi_{n}$. From condition (a) and Cauchy's inequality for derivatives we get

$$
\left|\xi_{n}\right| \leq \frac{3 ! \beta}{\left(\left(a-a_{0}\right) / 2\right)^{3}} \quad \text { for } n \geq 1 \text {. }
$$

Therefore we can find $\delta>0$ such that $\delta<\delta_{0}$ and for $|t| \leq \delta$,

$$
g_{n}^{\prime \prime}(t) \leq-(1 / 2) \text { for all } n \geq 1 \text {. }
$$

This verifies condition (2.29) of Theorem 2.6 and the proof is complete.

LEMMA 3.2. Let $\left\{T_{n}, n \geq 1\right\}$ be a sequence of random variables. Let $\left\{m_{n}\right\}$ be a bounded sequence of real numbers such that there exists a sequence $\left\{\tau_{n}\right\}$ satisfying (3.3). Let $d_{n}=\sqrt{a_{n} \psi_{n}^{\prime \prime}\left(\tau_{n}\right)}$ and $b_{n}=\tau_{n} d_{n}$ for $n \geq 1$. Suppose that $a_{n} \rightarrow \infty$ such that $\tau_{n} \sqrt{a_{n}} \rightarrow \infty$. Let the random variable $Y_{n}$ be as defined following identity (3.4). Suppose that $T_{n}$ satisfies conditions (a) and (b) and the following condition (c):

(c) There exists $\delta_{0}>0$ such that

$$
\sup _{\delta<|t| \leq \lambda \tau_{n}}\left|\frac{\phi_{n}\left(\tau_{n}+i t\right)}{\phi_{n}\left(\tau_{n}\right)}\right|=o\left(\frac{1}{\sqrt{a_{n}}}\right)
$$

for any given $\delta$ and $\lambda$ such that $0<\delta<\delta_{0}<\lambda$. Then

$$
b_{n} E\left(\exp \left(-b_{n} Y_{n}\right) I\left(Y_{n} \geq 0\right)\right) \rightarrow \frac{1}{\sqrt{2 \pi}} .
$$

Proof. Lemma 3.1 shows that $Y_{n}$ converges weakly to the standard normal and there exists a $\delta>0$ such that (2.7) holds with $\beta_{n}=\delta d_{n}$. Using condition (c) we get that for fixed $\lambda>0$,

$$
\begin{aligned}
\sup _{\delta d_{n}<|t| \leq \lambda b_{n}}\left|\hat{f}_{n}(t)\right| & =\sup _{\delta<|t| \leq \lambda \tau_{n}}\left|\frac{\phi_{n}\left(\tau_{n}+i t\right)}{\phi_{n}\left(\tau_{n}\right)}\right| \\
& =o\left(\frac{1}{\sqrt{a_{n}}}\right) \\
& =o\left(\frac{1}{b_{n}}\right)
\end{aligned}
$$

This verifies condition (2.8). The assertion (3.17) now follows from Theorem 2.3 and Theorem 2.7.

We are now in a position to state the main theorem of this section.

TheOREM 3.3. Let $\left\{T_{n}, n \geq 1\right\}$ be a sequence of random variables. Let $\left\{m_{n}\right\}$ be a bounded sequence of real numbers and $\left\{\tau_{n}\right\}$ be a sequence satisfying (3.3). 
Suppose that $a_{n} \rightarrow \infty$ such that $\tau_{n} \sqrt{a_{n}} \rightarrow \infty$. Assume that $T_{n}$ satisfies conditions (a), (b) and (c). Then

$$
P\left(\frac{T_{n}}{a_{n}} \geq m_{n}\right) \sim \frac{1}{\tau_{n} \sqrt{2 \pi a_{n} \psi_{n}^{\prime \prime}\left(\tau_{n}\right)}} \exp \left(-a_{n} \gamma_{n}\left(m_{n}\right)\right) .
$$

Proof. The conclusion (3.19) follows from Lemma 3.2 and the identity (3.5).

REMARK 3.4. We will now examine the class of lattice valued random variables $\left\{T_{n}\right\}$ satisfying conditions (a), (b) and (c). Let $T_{n}$ be a lattice valued random variable with span $p_{n}$ and $m_{n}$ be in the range of $T_{n} / a_{n}$. Then the random variable $Y_{n}$ is lattice valued with span $h_{n}=p_{n} / d_{n}$ and displacement 0 . The c.f. of $Y_{n}$ is periodic and its absolute value achieves the value 1 at multiples of $2 \pi / h_{n}$. When (a) and (b) hold, Lemma 3.1 shows that (3.6) holds, which implies that $p_{n}$ is bounded. Again, when (c) holds, $p_{n} \tau_{n} \rightarrow 0$ as $n \rightarrow \infty$. Theorem 3.5 considers the case where the $p_{n}$ 's and $\tau_{n}$ 's are bounded below by a positive number, and obtains the strong large deviation conclusion (3.20) by replacing condition (c) with condition $\left(\mathrm{c}^{\prime}\right)$. When $p_{n} \tau_{n} \rightarrow 0$ condition $\left(\mathrm{c}^{\prime}\right)$ implies condition (c) and the conclusion (3.20) of Theorem 3.5 agrees with the conclusion (3.19) of Theorem 3.3.

THEOREM 3.5. Let $T_{n}$ be a lattice valued random variable with span $p_{n}$. Let $m_{n}$ be in the range of $T_{n} / a_{n}$ and let $\left\{\tau_{n}\right\}$ be a sequence satisfying (3.3). Assume that $0<p^{*}<p_{n}<p^{* *}$ and $0<d<\tau_{n}<a_{0}<a$ for all $n \geq 1$. Let $T_{n}$ satisfy conditions (a) and (b) of Lemma 3.1 and the following condition $\left(\mathrm{c}^{\prime}\right)$ :

(c') There exists $\delta_{1}>0$, such that for $0<\delta<\delta_{1}$,

$$
\sup _{\delta<|t| \leq \pi / p_{n}}\left|\frac{\phi_{n}\left(\tau_{n}+i t\right)}{\phi_{n}\left(\tau_{n}\right)}\right|=o\left(\frac{1}{\sqrt{a_{n}}}\right) .
$$

Then

$$
P\left(\frac{T_{n}}{a_{n}} \geq m_{n}\right) \sim \frac{p_{n}}{\sqrt{2 \pi a_{n} \psi_{n}^{\prime \prime}\left(\tau_{n}\right)}} \frac{\exp \left(-a_{n} \gamma_{n}\left(m_{n}\right)\right)}{\left(1-\exp \left(-\tau_{n} p_{n}\right)\right)} .
$$

PRoof. Let $Y_{n}$ be as defined following identity (3.4). Then $Y_{n}$ is a lattice valued random variable with span $h_{n}=p_{n} / d_{n}$ and displacement 0 . Let $b_{n}=$ $\tau_{n} d_{n}$. Since $p_{n}$ and $\tau_{n}$ are bounded above and below by positive numbers we have $0<\liminf _{n} b_{n} h_{n}<\infty$. Lemma 3.1 shows that $Y_{n}$ converges weakly to the standard normal and there exists a $\delta>0$ such that (2.40) holds with $\beta_{n}=\delta d_{n}$. Using condition ( $\left.\mathrm{c}^{\prime}\right)$ we can easily verify that $Y_{n}$ satisfies $(2.41)$. The conclusion (3.20) now follows from Theorems 2.9 and 2.10.

REMARK 3.6. Bahadur and Ranga Rao (1960) obtained a strong large deviation result for sums of i.i.d. random variables, which included a result of 
Blackwell and Hodges (1959) in the lattice case. We will now show that the result of Bahadur and Ranga Rao (1960) can be obtained from our Theorems 3.3 and 3.5. Let $X_{1}, X_{2}, \ldots$ be i.i.d. nondegenerate random variables with m.g.f. $\phi(z)$ and let $\psi(z)=\log (\phi(z))$, be finite for $|z|<a$. Let $T_{n}=X_{1}+$ $\cdots+X_{n}$. The m.g.f. of $T_{n}$ is given by $\phi_{n}(z)=\phi^{n}(z)$. Let $m$ be a real number such that there exists $0<\tau<a$ satisfying $\psi^{\prime}(\tau)=m$. Let $m_{n}=m$ and $a_{n}=n$ for all $n \geq 1$. Conditions (a) and (b) are trivially satisfied since $\psi_{n} \equiv \psi$ and $\tau_{n}=\tau$ for all $n \geq 1$.

If $X_{1}$ is nonlattice valued, then $\phi(\tau+i t) / \phi(\tau)$ is the c.f. of a nonlattice valued random variable and hence its supremum on an compact interval not containing 0 is less than 1 . This verifies condition (c). Let $\gamma(m)=\sup _{s}[m s-$ $\psi(s)]$. The strong large deviation result

$$
P\left(\frac{T_{n}}{n} \geq m\right) \sim \frac{1}{\tau \sqrt{2 \pi n \psi^{\prime \prime}(\tau)}} \exp (-n \gamma(m))
$$

follows from Theorem 3.3. Bahadur and Ranga Rao (1960) established (3.21) by considering separately the two cases where $X_{1}$ satisfies Cramér's condition and where $X_{1}$ is nonlattice and does not satisfy Cramér's condition. The above application of Theorem 3.3 shows that it is not necessary to separate these two cases.

If $X_{1}$ is lattice valued random variable with span $p$, then $\phi(\tau+i t) / \phi(\tau)$ is the c.f. of a random variable with span $p$. It follows that

$$
\sup _{\delta<|t| \leq \pi / p}\left|\frac{\phi(\tau+i t)}{\phi(\tau)}\right|<1,
$$

for any $\delta>0$. This verifies condition $\left(\mathrm{c}^{\prime}\right)$. The strong large deviation result

$$
P\left(\frac{T_{n}}{n} \geq m\right) \sim \frac{p}{\sqrt{2 \pi n \psi^{\prime \prime}(\tau)}} \frac{\exp (-n \gamma(m))}{(1-\exp (-\tau p))}
$$

follows from Theorem 3.5. Thus we have verified that all cases of Theorem 1 of Bahadur and Ranga Rao (1960) follow from our Theorems 3.3 and 3.5.

4. Applications. In this section we give two typical applications to illustrate the large deviation limit theorems and strong large deviation limit theorems of the previous sections. The first example is a local limit result and illustrates Theorem 2.1. The second example is a strong large deviation result for a lattice valued random variable and illustrates Theorem 3.3.

EXAMPLE 4.1. This example applies to a general class of sums of dependent random variables considered in Chaganty and Sethuraman (1987). Though it was proved in that paper that the limit distribution could be both normal and nonnormal, our example applies only to the case where the limit distribution is normal. We first present a particular application and then state a more general application referring to conditions found in Chaganty and Sethuraman (1987). 
Let $\left\{X_{1}^{(n)}, X_{2}^{(n)}, \ldots, X_{n}^{(n)}\right\}$ be a triangular array of random variables with joint density function

$$
d Q_{n}^{*}(\mathbf{x})=z_{n}^{-1}(2 \pi)^{-n / 2}\left[\cosh \left(\frac{s_{n}}{\beta n}\right)\right]^{n} \exp \left(-\sum_{j=1}^{n} \frac{x_{j}^{2}}{2}\right) d \mathbf{x},
$$

where $\mathbf{x}=\left(x_{1}, \ldots, x_{n}\right), s_{n}=x_{1}+\cdots+x_{n}, \beta>1$ and $z_{n}$ is a normalizing constant. Such dependent random variables arise in generalized Curie-Weiss models used to describe ferromagnets. Using Theorem 3.7 of Chaganty and Sethuraman (1987) or using (4.3) we can show that $Y_{n}=\left(X_{1}^{(n)}+\right.$ $\left.\cdots+X_{n}^{(n)}\right) / \sqrt{n}$ converges in distribution to a normal distribution with mean 0 and variance $\sigma^{2}=\beta^{2} /\left(\beta^{2}-1\right)$ [Example 4.4 of Chaganty and Sethuraman (1987) considered the case $\beta=1$ and obtained a nonnormal distribution under a different normalization]. We will now show that Theorem 2.1 applies to $Y_{n}$. Since

$$
(\cosh \omega)^{n}=\sum_{y \in C_{n}} \exp (\omega y) \lambda_{n}(y)
$$

with $\lambda_{n}(y)=\left(\begin{array}{c}n \\ (n+y) / 2\end{array}\right) 2^{-n}$ and $C_{n}=\{-n,-n+2, \ldots, n\}$, the c.f. of $Y_{n}$ is given by

$$
\begin{aligned}
\hat{f}_{n}(t) & =E\left(\exp \left(i t Y_{n}\right)\right) \\
& =z_{n}^{-1} \sum_{y \in C_{n}}\left[\frac{1}{(2 \pi)^{n / 2}} \int \exp \left(\frac{i t s_{n}}{\sqrt{n}}+\frac{y s_{n}}{\beta n}-\sum_{j=1}^{n} \frac{x_{j}^{2}}{2}\right) d \mathbf{x}\right] \lambda_{n}(y) \\
& =\exp \left(-\frac{t^{2}}{2}\right) z_{n}^{-1} \sum_{y \in C_{n}} \exp \left(\frac{i t y}{\beta \sqrt{n}}+\frac{y^{2}}{2 \beta^{2} n}\right) \lambda_{n}(y) .
\end{aligned}
$$

Since $\hat{f}_{n}(0)=1$, we have

$$
\left|\hat{f}_{n}(t)\right| \leq \exp \left(-t^{2} / 2\right) \text { for all } n \text { and } t .
$$

Thus from Theorem 2.1 we get, if $y_{n} \rightarrow y$,

$$
f_{n}\left(y_{n}\right) \rightarrow \frac{1}{\sqrt{2 \pi} \sigma} \exp \left(-\frac{y^{2}}{2 \sigma^{2}}\right)
$$

where $f_{n}(y)$ is the p.d.f. of $Y_{n}$ and $\sigma^{2}=\beta^{2} /\left(\beta^{2}-1\right)$.

From the preceding discussion and from a full use of Theorem 3.7 of Chaganty and Sethuraman (1987) we have the following application which we state without proof.

Let $\left\{X_{1}^{(n)}, X_{2}^{(n)}, \ldots, X_{n}^{(n)}\right\}$ be a triangular array of random variables whose joint distribution is as given in (3.13) of Theorem 3.7 of Chaganty and Sethuraman (1987). We will impose conditions on the probability measure $P$ and the index $r$ appearing in that theorem. Let $P$ be the standard normal distribution and let $r=1$. Under these conditions, Theorem 3.7 of Chaganty 
and Sethuraman (1987) shows that there is a sequence of constants $\left\{m_{n}\right\}$ such that

$$
Y_{n}=\left(\sum_{j=1}^{n} X_{i}^{(n)}-n m_{n}\right) / \sqrt{n}
$$

has a limiting normal distribution with mean 0 and variance $\sigma^{2}$. Let $\hat{f}_{n}(t)$ be the c.f. of $Y_{n}$. For this case, if we proceed as in the previous application, we can establish (4.4) for all $n$ and $t$. This shows that (4.5) is true with the appropriate $\sigma$.

EXAMPLE 4.2. We now obtain a strong large deviation result for the Wilcoxon signed-rank statistic under the null hypothesis. This strengthens the well-known weak large deviation results for this statistic [see Klotz (1965)].

Let $\left\{X_{n}, n \geq 1\right\}$ be a sequence of i.i.d. continuous random variables symmetric about their median $m$. Arrange $\left|X_{1}\right|,\left|X_{2}\right|, \ldots,\left|X_{n}\right|$ in increasing order of magnitude and assign ranks $1,2, \ldots, n$. The Wilcoxon signed-rank statistic $W_{n}$ is defined as the sum of the ranks of positive $X_{i}$ 's. The statistic $W_{n}$. is used to test the null hypothesis $H_{0}: m=0$ versus $H_{1}: m \neq 0$. Let $T_{n}=U_{n} / n$. The random variable $T_{n}$ is a lattice random variable with span $p_{n}=1 / n$. The m.g.f. of $T_{n}$ under the null hypothesis $H_{0}$ is given by

$$
\phi_{n}(z)=\prod_{k=1}^{n}[(\exp (k z / n)+1) / 2], \quad z \in \mathscr{C} .
$$

It is easy to check that $\phi_{n}(z)$ is analytic and nonvanishing in the region $\Omega=\{z \in \mathscr{b}:|z|<\pi / 2\}$. Let

$$
\psi_{n}(z)=n^{-1} \log \phi_{n}(z) .
$$

It is easy to check that there exists $\beta>0$ such that $\left|\psi_{n}(z)\right|<\beta$ for $|z|<\pi / 2$. Straightforward calculations show that $\psi_{n}^{\prime \prime}(\tau)$ is bounded below by a positive number $\alpha$ for real $\tau$ such that $|\tau|<\pi / 2$. Thus $T_{n}$ satisfies conditions (a) and (b). Next we first note that $\psi_{n}^{\prime}(s) \rightarrow \int_{0}^{1}(x) /(1+\exp (-s x)) d x$ and that the range of $\psi_{n}^{\prime}(s)$ for real $s$ contains the open interval $(0,1 / 2)$ for all $n \geq 1$. Thus if $\left\{m_{n}\right\}$ is a sequence of real numbers such that $1 / 4<m_{n}<\bar{m}<\int_{0}^{1}(x) /(1+$ $\exp (-\pi x / 2)) d x$, then we can find a positive number $a_{0}$ and a sequence $\left\{\tau_{n}\right\}$ satisfying $0<\tau_{n}<a_{0}<\pi / 2$ and $\psi_{n}^{\prime}\left(\tau_{n}\right)=m_{n}$, for all $n \geq 1$. From the analysis in Example 3.1 of Chaganty and Sethuraman (1985) it can be seen that there exists $n_{0}$ and $\delta_{1}>0$ such that for $0<\delta<\delta_{1}$,

$$
\sup _{\delta<|t| \leq \pi / p_{n}}\left|\frac{\phi_{n}\left(\tau_{n}+i t\right)}{\phi_{n}\left(\tau_{n}\right)}\right| \leq \exp \left(-\frac{n \alpha \delta^{2}}{4}\right)
$$

for $n \geq n_{0}$. Since $p_{n} \rightarrow 0$ this verifies condition (c). Therefore Theorem 3.3 shows that the conclusion (3.19) holds.

Acknowledgment. We are very grateful to a referee for carefully reading the original version of this paper and for suggesting substantial improvements. 


\section{REFERENCES}

Apostol, T. M. (1974). Mathematical Analysis. Addison-Wesley, Reading, MA.

BAHADUR, R. R. and RANGA RAO, R. (1960). On deviations of the sample mean. Ann. Math. Statist. 31 1015-1027.

Blackwell, D. and Hodges, J. L. (1959). The probability in the extreme tail of a convolution. Ann. Math. Statist. 30 1113-1120.

Chaganty, N. R. and Sethuraman, J. (1985). Large deviation local limit theorems for arbitrary sequences of random variables. Ann. Probab. 13 97-114.

Chaganty, N. R. and Sethuraman, J. (1986). Multi-dimensional large deviation local limit theorems. J. Multivariate Anal. 20 190-204.

Chaganty, N. R. and Sethuraman, J. (1987). Limit theorems in the area of large deviations for some dependent random variables. Ann. Probab. 15 628-645.

Chernoff, H. (1952). A measure of asymptotic efficiency for tests of an hypothesis based on the sum of observations. Ann. Math. Statist. 23 493-507.

Cramér, H. (1938). Sur un nouveau théorème-limite de la théorie des probabilitès. Actualites Sci. Ind. 736 5-23.

De HaAn, L. and Resnick, S. I. (1982). Local limit theorems for sample extremes. Ann. Probab. 10 396-413.

Ellis, R. S. (1984). Large deviations for a general class of dependent random vectors. Ann. Probab. 12 1-12.

Feller, W. (1967). On regular variation and local limit theorems. Proc. Fifth Berkeley Symp. Math. Statist. Probab. 2 373-388. Univ. California Press, Berkeley.

JAIN, N. C. and PRUITT, W. E. (1987). Lower tail probability estimates for subordinators and nondecreasing random walks. Ann. Probab. 15 75-101.

KцOTZ, J. (1965). Alternative efficiencies for signed rank tests. Ann. Math. Statist. 36 1759-1766. Sievers, G. L. (1969). On the probability of large deviations. Ann. Math. Statist. 40 1908-1921.

Steinebach, J. (1978). Convergence rates of large deviation probabilities in the multidimensional case. Ann. Probab. 6 751-759.

VARAdhan, S. R. S. (1984). Large Deviations and Applications. SIAM, Philadelphia.

Department of Mathematics and Statistics

OLD DOMINION UNIVERSITY

NORFOLK, VIRGINIA 23529
Department of STAtistics

Florida State University

TAllahassee, Florida 32306 